'D Grenier, ${ }^{2} \mathrm{AM}$ Ugnat, ${ }^{3} \mathrm{MA}$ Davis, ${ }^{1} \mathrm{M}$ Laffin Thibodeau. ${ }^{1}$ Canadian Paediatric Surveillance Program; ${ }^{2}$ Public Health Agency of Canada; ${ }^{3}$ Canadian Paediatric Society, Ottawa, ON, Canada

Background Paediatric neurological diseases individually are rare; however, collectively affect thousands of children and have life-long impacts. The incidence of many of these is not readily available, and yet essential for improving clinical care, advocacy and health service planning.

Aims To obtain/examine national population-based data, in a timely manner, on acute flaccid paralysis (AFP), progressive intellectual and neurological deterioration (PIND), acquired demyelinating syndromes of the central nervous system (ADS), congenital myotonic dystrophy (CMD) and paediatric myasthenia (PM).

Methods Studies were conducted through the Canadian Paediatric Surveillance Program, a network of $>2,500$ paediatricians, reporting cases monthly according to preset protocols. Confidentiality is mandatory; studies receive ethical approval.

Results The AFP study, with 657 cases in 15 years, affirms that Canada is free of wild-type poliovirus. The PIND study demonstrated several genetically defined neurodegenerative disorders, and only one case of iatrogenic Creutzfeldt-Jakob disease. A yearly incidence of 0.9 per 100,000 was estimated to affect Canadian children during the ADS study, with optic neuritis being the most common presentation. Awareness of multiple sclerosis as a possible outcome of ADS increased remarkedly. Of 38 confirmed CMD cases in six years, $61 \%$ were index cases for families. In year one of surveillance, 33 cases of PM were confirmed; almost half not having elevated titers of acetylcholine receptor antibodies, and $21 \%$ having other co-existing or familial immune disorders.

Conclusion Active national surveillance has more reliably characterized several rare neurological disorders and their associated burdens, supporting and informing the development of medical and public health interventions.

\section{THE IMPORTANCE OF SECRETORY IGA ON TRANS- EPITHELIAL TRANSPORT OF COMMENSAL BACTERIA AND ITS CONSEQUENCES ON NEONATAL IMMUNE DEVELOPMENT: PRECLINICAL ASSESSMENT}

doi:10.1136/archdischild-2012-302724.0053

${ }^{1} \mathrm{~L}$ Favre, ${ }^{2} \mathrm{~N}$ Rol, ${ }^{1} \mathrm{~B}$ Reuge, ${ }^{2} \mathrm{~B}$ Corthésy, ${ }^{1} \mathrm{~J}$ Benyacoub. 'Nutrition \& Health, Nestlé Research Center; 'Immunology \& Allergy, State University Hospital, Lausanne, Switzerland

Background and aims Secretory $\operatorname{IgA}$ (SIgA) naturally binds to commensal bacteria and is able to cross back through the intestinal epithelium to promote immune responses. The aim of the present work was to investigate the contribution of SIgA on the trans-epithelial transport of commensal bacteria, such as probiotics, and its consequences on neonatal immune development as such process may also occur with SIgA originating from breast milk.

Methods A) Fluorescent bacteria alone or associated with SIgA as immune complexes (IC) were administered into intestinal loops containing one Peyer's patch (PP) in SPF or germ-free mice. Fate of bacteria within PP over time was analyzed by confocal microscopy.

B) After day 7 of life, germ-free mouse neonates were conventionalized to induce natural neonatal gut colonization and supplemented up to weaning with either placebo, probiotics or IC. Immune maturation was then assessed by measuring mucosal IgA production (ELISPOTs) in PPs.

\section{Results}

a. Natural entry of commensal bacteria into PP was speeded up when administered in the form of IC. In germ-free mice, lacking endogenous SIgA, a very low level of trans-epithelial transport of commensal bacteria was observed, which was restored with IC b. While early-life supplementation with probiotics alone significantly enhanced occurrence of IgA producing cells in PPs of pups as compared to controls, IC feeding significantly further increased it.

Conclusions SIgA-mediated entry of commensal bacteria in PPs represents a mechanism ensuring the continuous dialogue between the host and its microbiota, particularly relevant for neonatal immune development.

\section{CEACAM1 IN BRAIN DEVELOPMENT AND PRETERM BRAIN INJURY}

doi:10.1136/archdischild-2012-302724.0054

${ }^{1,2} \mathrm{~S}$ Prager, ${ }^{2 B B}$ Singer, ${ }^{1} M$ Keller, ${ }^{1}$ B Bendix, 'GW Schlager, ${ }^{1} \mathrm{R}$ Herrmann, ${ }^{3} \mathrm{~S}$ Ergün, 'U Felderhoff-Müser. 'Department of Pediatrics 1, Neonatology; 'Institute of Anatomy,

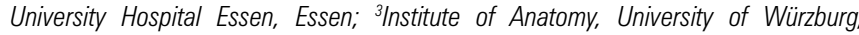
Würzburg, Germany

Background and aims About $10 \%$ of all neonates are born preterm before 37 weeks of gestation. These babies are at high risk to develop morbidities such as neurocognitive disorders (encephalopathy of prematurity) which are a huge burden on the children and their families. Multiple factors are causal, e.g. hyperoxia and maternal/neonatal inflammation. We demonstrated that CEACAM1, a member of the carcinoembryonic antigen-related cell adhesion molecule (CEACAM) family, is expressed ontogenetically in oligodendrocytes of the developing brain. Since CEACAM1 is involved in inflammation-associated signaling we hypothesize that CEACAM1 might contribute to inflammationinduced preterm brain injury.

Material and Methods In a rat model of inflammation-induced encephalopathy of prematurity (LPS at p3), changes in CEACAM1 expression were quantified on RNA level at $\mathrm{p} 6$ and $\mathrm{p} 11$. Animals were anesthetized, transcardially perfused, and forebrains were immediately removed and snap-frozen. RNA from forebrains was isolated according to standard protocols. CEACAM1 isoform expression was quantified by qRT-PCR.

Results LPS exposure at p3 induces significant changes in CEACAM1 expression in the developing brain. We report a significant increase of the soluble isoform CEACAM1-4C2 at p6, and a subsequent increase of the CEACAM1-4L isoform and an isoform shift from CEACAM1-4S towards CEACAM1-4L at p11.

Conclusions Although underlying mechanisms are still elusive we demonstrate that CEACAM1 expression in oligodendrocytes is significantly altered in a model of inflammation-induced encephalopathy of prematurity. This finding emphasizes our hypothesis that CEACAM1 is involved in detrimental processes in the immature brain.

\section{ADVERSE EVENTS FOLLOWING THE COMBINATION OF DEXMEDETOMIDINE WITH THERAPEUTIC HYPOTHERMIA IN A PIGLET ASPHYXIA MODEL}

doi:10.1136/archdischild-2012-302724.0055

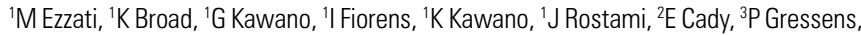
${ }^{4} \mathrm{R}$ Sanders, ${ }^{5} \mathrm{X}$ Golay, ${ }^{1} \mathrm{NJ}$ Robertson. ${ }^{1}$ Institute for Women's Health, University College London; ${ }^{2}$ Medical Physics and Bioengineering, University College Hospitals, London, UK: ${ }^{3}$ Inserm, Paris, France; ${ }^{4}$ Anesthetics, Imperial College London; ${ }^{5}$ Brain Repair and Rehabilitation, Institute of Neurology, London, UK

Background Dexmedetomidine (DXM) is a potent, selective a2 adrenoceptor agonist which exerts sedative, neuroprotective, analgesic and anti-inflammatory properties that may be beneficial for neonatal asphyxia. The safety of DXM combined with therapeutic hypothermia is unknown.

Aim To assess safety of low $(0.6-1.5 \mathrm{mcg} / \mathrm{kg} / \mathrm{h})$ and high dose $(10 \mathrm{mcg} / \mathrm{kg} / \mathrm{h})$ DXM with hypothermia as part of a larger study investigating neuroprotection with DXM-augmented cooling. 